\title{
Effect of small-dose levosimendan on mortality rates and organ functions in Chinese elderly patients with sepsis
}

This article was published in the following Dove Press journal:

Clinical Interventions in Aging

29 May 2017

Number of times this article has been viewed

\author{
Xin Wang ${ }^{1, *}$ \\ Shikui $\mathrm{Li}^{2, *}$ \\ 'Intensive Care Unit, ${ }^{2}$ Cardiothoracic \\ Surgery, Daqing Oilfield General \\ Hospital, Daqing, Heilongjiang, \\ People's Republic of China \\ *These authors contributed equally \\ to this work
}

Aim: As a primary cause of death not only in Western countries but also in the People's Republic of China, sepsis is diagnosed as abnormal organ functions as a result of a disordered response to a severe infection. This study was designed to assess the effect of small-dose levosimendan without a loading dose on mortality rates and organ functions in Chinese elderly patients with sepsis.

Methods: Following a prospective, randomized, and double-blinded design, 240 Chinese elderly patients with sepsis shock were admitted to the intensive care unit (ICU). All patients were randomly and evenly assigned into a levosimendan group (number of patients $=120$ ) and a control group (number of patients $=120$ ). The control group underwent standard care, and the levosimendan group was administered levosimendan in addition to standard care.

Results: All participants, comprising 134 males (55.8\%) and 106 females (44.2\%), were 70 (67-73) years old. Baseline characteristics, preexisting illnesses, initial infections, organ failures, and additional agents and therapies showed no significant difference between the two groups ( $P>0.05$ for all). There were no significant differences in mortality rates at 28 days, at ICU discharge, and at hospital discharge between the two groups ( $P>0.05$ for all). The number of days of ICU and hospital stay in the levosimendan group was significantly less than for those in the control group $(P<0.05$ for all). Mean daily total sequential organ failure assessment score and all organ scores except the cardiovascular scores showed no significant difference between the two groups ( $P>0.05$ for all). Cardiovascular scores in the levosimendan group were significantly higher than those in the control group $(P<0.05$ for all).

Conclusion: Small-dose levosimendan could not reduce the mortality rates or enhance the respiratory, liver, renal, and coagulation functions, but could shorten the days of ICU and hospital stay, and improve the cardiovascular function, which suggests that small-dose levosimendan is valuable for Chinese elderly patients with sepsis.

Keywords: Chinese elderly, levosimendan, mortality rate, organ function, sepsis, small-dose

\section{Introduction}

Patients with sepsis have a mortality rate of $30 \%-40 \%$ across the world. ${ }^{1}$ As a primary cause of death not only in Western countries but also in China, sepsis is diagnosed as abnormal organ functions as a result of a disordered response to a severe infection. ${ }^{2}$ Its most severe appearance is septic shock because of circulatory disturbance and metabolic abnormalities. ${ }^{3}$ Levosimendan (Qilu Pharmaceutical, Jinan, People's Republic of China) is a new kind of calcium-sensitizing agent that has the potential to improve the cardiovascular function and blood circulation without obviously increasing the oxygen demand and impairing the diastolic relaxation. ${ }^{4-6}$ Moreover, previous studies have suggested that levosimendan improves the pulmonary, renal, liver, and
Correspondence: Shikui Li Cardiothoracic Surgery, Daqing Oilfield General Hospital, 8 Zhongkang Street, Saertu District, Daqing I6300I, Heilongjiang, People's Republic of China Email iskxw12@163.com 
coagulation functions. ${ }^{7-9}$ Clinical data on the application of levosimendan in Chinese patients with sepsis have been scarce and controversial, and it is essential to perform a prospective study to evaluate the curative value of levosimendan in Chinese elderly patients with sepsis. ${ }^{10}$ Besides, a recent study that investigated the effect of levosimendan at an infusion rate of $0.2-0.4 \mu \mathrm{g} / \mathrm{kg} / \mathrm{min}$ or with a loading dose did not reach a satisfactory conclusion. ${ }^{11}$ The present study was designed to assess the effect of small-dose levosimendan $(0.1-0.2 \mu \mathrm{g} / \mathrm{kg} / \mathrm{min})$ without a loading dose on mortality rates and organ functions in Chinese elderly patients with sepsis.

\section{Methods}

\section{Participants and procedures}

Following a prospective, randomized, and double-blinded design, 240 Chinese patients 65 years old or older with septic shock were admitted to the intensive care unit (ICU) between June 2012 and December 2016. Septic shock was diagnosed on the basis of the following criterion: patients with agents to maintain the mean arterial pressure of at least $65 \mathrm{mmHg}$ in spite of appropriate volume resuscitation. The exclusion criteria were pregnancy, significant valvular heart disease, present or suspected acute coronary syndrome, and limitations to the use of inotropes such as ventricular outflow tract obstruction and mitral valve systolic anterior motion (Figure 1). The study protocol was approved by the Ethics Committee of Daqing Oilfield General Hospital. Each participant provided written informed consent to be included in the study. All patients were randomly and evenly assigned into a levosimendan group (number of patients $=120$ ) and a control group (number of patients $=120$ ) using computer-generated random numbers in a randomized block design. The control group underwent standard care, and the levosimendan group was administered levosimendan (Specifications: $5 \mathrm{~mL}, 12.5 \mathrm{mg}$ ) at a continuous intravenous infusion rate of $0.1-0.2 \mu \mathrm{g} / \mathrm{kg} / \mathrm{min}$ without a loading dose for 24 hours in addition to standard care.

\section{Follow-up and end points}

The mean daily sequential organ failure assessment (SOFA) Score of patients in ICU was the end point followed up from randomization to a maximum of 28 days. ${ }^{12}$ The daily SOFA score was calculated for each patient on the basis of five organ scores: cardiovascular, respiratory, liver, renal, and coagulation functions (scores for each organ range from 0 to 4 , with higher scores indicating more severe abnormality of organ functions; maximum score, 20). Because of the difficulty in accurately scoring the Glasgow Coma Score daily in the presence of sedation, the Glasgow Coma Score was not recommended by previous studies ${ }^{13,14}$ and was not applied in the present study. Daily scores were totaled and divided by the number of days of ICU stay in order to calculate the mean daily SOFA score. Not only the mortality rates at 28 days, at ICU discharge, and at hospital discharge, but also the number of days of ICU and hospital stay, were recorded and compared between the two groups. All patients continually received the assigned agents and were not lost during the follow-up.

\section{Description and statistics}

Continuous variables with normal distribution were reported using mean and standard deviation, and the difference between the two groups was compared using Student's $t$-test. Continuous variables with abnormal distribution were reported using median and interquartile range, and the difference between the two groups was compared using the

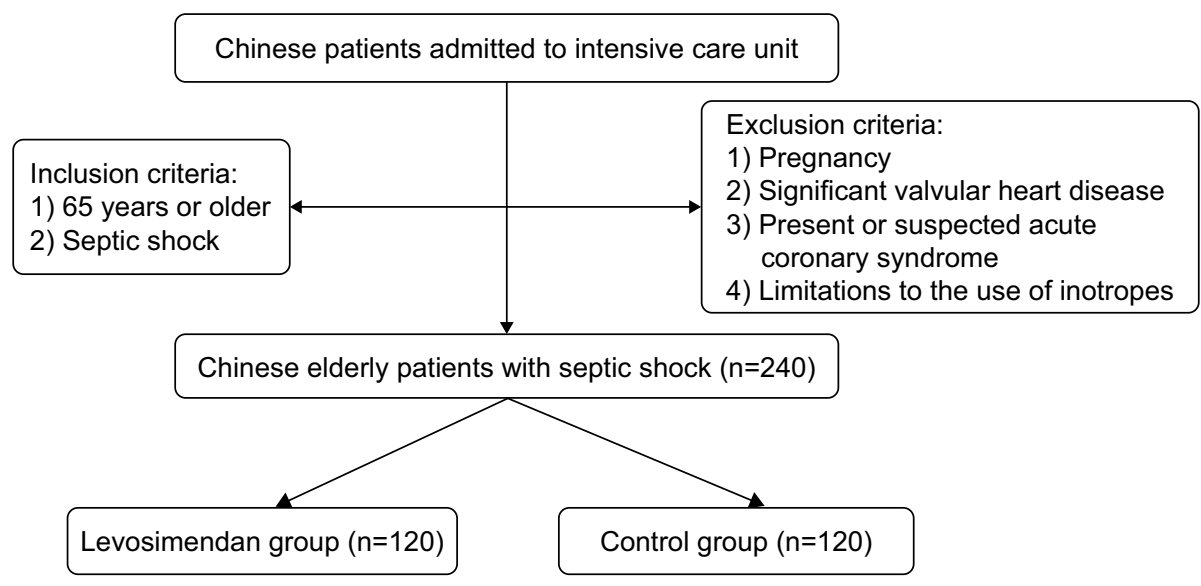

Figure I Protocol, inclusion and exclusion criteria.

Abbreviation: n, number of patients. 
Mann-Whitney U test. Categorical variables were reported with number and percentage, and the difference between the two groups was compared with the $\chi^{2}$ test. Statistical analyses were carried out by the Statistical Package for Social Science (SPSS) version 17.0 software (SPSS Inc., Chicago, IL, USA), and a $P$-value $<0.05$ was accepted as statistically significant.

\section{Results}

Participants, comprising 134 males (55.8\%) and 106 females (44.2\%), were 70 (67-73) years old. As shown in Table 1, baseline characteristics, preexisting illnesses, initial infections, organ failures, and additional agents and therapies showed no significant difference between the two groups $(P>0.05$ for all). As shown in Table 2, there were no significant differences in mortality rates at 28 days, at ICU discharge, and at hospital discharge between the two groups ( $P>0.05$ for all). The number of days of ICU and hospital stay was significantly less in the levosimendan group than in the control group ( $P<0.05$ for all). The mean daily total SOFA score and all organ scores except the cardiovascular scores showed no significant difference between the two groups ( $P>0.05$ for all). Cardiovascular scores in the levosimendan group were significantly higher than those in the control group $(P<0.05$ for all $)$.

\section{Discussion}

As a new kind of calcium-sensitizing agent, levosimendan has the ability to improve the cardiovascular function through the selective combination with myocardial troponin $\mathrm{C}$, and the effective stabilization of $\mathrm{Ca}^{2+}$-dependent interaction between cardiac troponin I and cardiac troponin T. ${ }^{4}$ Levosimendan can be combined with cardiac troponin $\mathrm{C}$ zsitivity to $\mathrm{Ca}^{2+}$, leading to myocardial contraction even at the same or lower $\mathrm{Ca}^{2+}$ concentration. ${ }^{15}$ Levosimendan has no obvious effect on oxygen demand

Table I Baseline characteristics of patients with sepsis in the levosimendan and control groups

\begin{tabular}{|c|c|c|c|}
\hline Characteristics & $\begin{array}{l}\text { Levosimendan group } \\
(n=\mid 20)\end{array}$ & $\begin{array}{l}\text { Control group } \\
(n=\mid 20)\end{array}$ & $P$-value \\
\hline $\begin{array}{l}\text { Age (years), median } \\
\text { (interquartile range) }\end{array}$ & \multicolumn{3}{|c|}{ (interquartile range) } \\
\hline Males, n (\%) & $69(57.5)$ & $65(54.2)$ & 0.603 \\
\hline \multicolumn{4}{|l|}{ Preexisting illnesses, n (\%) } \\
\hline Ischemic heart disease & $18(15.0)$ & $13(10.8)$ & 0.336 \\
\hline Heart failure & II (9.2) & $9(7.5)$ & 0.640 \\
\hline Severe COPD & $12(10.0)$ & $9(7.5)$ & 0.493 \\
\hline Chronic renal failure & $12(10.0)$ & $13(10.8)$ & 0.833 \\
\hline Cirrhosis & $5(4.2)$ & $3(2.5)$ & 0.719 \\
\hline Diabetes & $32(26.7)$ & $27(22.5)$ & 0.454 \\
\hline Recent surgery & $41(34.2)$ & 47 (39.2) & 0.422 \\
\hline Initial infections, n (\%) & & & 0.533 \\
\hline Lung & $51(42.5)$ & $44(36.7)$ & \\
\hline Abdomen & $39(32.5)$ & $43(35.8)$ & \\
\hline Urinary tract & $8(6.7)$ & $13(10.8)$ & \\
\hline Soft tissue or catheter & $3(2.5)$ & $2(1.7)$ & \\
\hline Neurologic site & $2(1.7)$ & $6(5.0)$ & \\
\hline Primary bacteremia & $6(5.0)$ & $5(4.2)$ & \\
\hline Other & II (9.2) & $7(5.8)$ & \\
\hline \multicolumn{4}{|l|}{ Organ failures, n (\%) } \\
\hline Respiratory & $51(42.5)$ & $49(40.8)$ & 0.793 \\
\hline Liver & $5(4.2)$ & $7(5.8)$ & 0.554 \\
\hline Renal & $37(30.8)$ & $41(34.2)$ & 0.581 \\
\hline Neurologic & $59(49.2)$ & $56(46.7)$ & 0.698 \\
\hline Coagulation & $9(7.5)$ & $7(5.8)$ & 0.605 \\
\hline \multicolumn{4}{|c|}{ Additional agents and therapies, $\mathrm{n}(\%)$} \\
\hline Epinephrine & II (9.2) & $12(10.0)$ & 0.826 \\
\hline Norepinephrine & $63(52.5)$ & $59(49.2)$ & 0.606 \\
\hline Dopamine & $74(6 I .7)$ & 71 (59.2) & 0.692 \\
\hline Dobutamine & $19(15.8)$ & $24(20)$ & 0.400 \\
\hline Mechanical ventilation & $89(74.2)$ & $83(69.2)$ & 0.390 \\
\hline Renal replacement therapy & $21(17.5)$ & $25(20.8)$ & 0.512 \\
\hline
\end{tabular}

Abbreviation: $n$, number of patients. 
Table 2 Mortality rates and organ functions of patients with sepsis in the levosimendan and control groups

\begin{tabular}{|c|c|c|c|}
\hline Outcomes & $\begin{array}{l}\text { Levosimendan } \\
\text { group } \\
(\mathrm{n}=120)\end{array}$ & $\begin{array}{l}\text { Control } \\
\text { group } \\
(n=\mid 20)\end{array}$ & $P$-value \\
\hline \multicolumn{4}{|l|}{ Mortality rates, n (\%) } \\
\hline At 28 days & $33(27.5)$ & $39(32.5)$ & 0.398 \\
\hline At ICU discharge & $24(20.0)$ & $28(23.3)$ & 0.531 \\
\hline At hospital discharge & $36(30.0)$ & $44(36.7)$ & 0.273 \\
\hline Intensive care unit stay, days & $17(13-22)$ & $20(14-23)$ & 0.034 \\
\hline Hospital stay, days & $24(22-30)$ & $28(22-3 \mid)$ & 0.042 \\
\hline $\begin{array}{l}\text { Mean daily total sequential } \\
\text { organ failure assessment score }\end{array}$ & $8(0-12)$ & $8(2-13)$ & 0.249 \\
\hline Cardiovascular & $2(0-3)$ & $2(I-3)$ & 0.046 \\
\hline Respiratory & $2(0-3)$ & $2(1-3)$ & 0.208 \\
\hline Liver & I (0-2) & I $(0-2)$ & 0.332 \\
\hline Renal & $2(0-2)$ & $2(0-3)$ & 0.366 \\
\hline Coagulation & I (0-2) & I $(0-2)$ & 0.402 \\
\hline
\end{tabular}

Abbreviations: ICU, intensive care unit; $n$, number of patients.

and diastolic relaxation without disturbing the metabolism of cyclic adenosine monophosphate. ${ }^{5}$ By sensitizing cardiomyocytes to existing levels of intracellular calcium, an increase in the force of myocardial contraction is achieved with a minimal increase in myocardial oxygen demand. ${ }^{16}$ Previous studies have suggested that levosimendan has the potential to provide the beneficial cardioprotective and hemodynamic effects not only in the cardiovascular field but also in patients with sepsis. ${ }^{17}$ The treatment of sepsis with levosimendan should be specially considered in Chinese elderly patients. However, few researchers have paid attention to the curative value of levosimendan, and there have been few and controversial studies on the application of levosimendan in Chinese patients with sepsis. In the present study, although levosimendan could not reduce the mortality rates, it succeeded in shortening the days of ICU and hospital stay in Chinese elderly patients with sepsis. Sepsis is a complicated inflammation caused by infection, and with levosimendan, it is difficult to increase the survival of patients with sepsis. Hence, this is not a surprising result in the present study. Combining levosimendan with antiinflammatory compounds and supportive treatments may offer a higher chance of curing the patients and improving their survival.

Meanwhile, previous studies have shown a potential benefit of levosimendan on cardiovascular, pulmonary, renal, hepatic, and coagulation functions in patients with sepsis. ${ }^{6-9}$ As an inotropic agent, levosimendan has also appeared to play anti-inflammatory, antioxidative, and antiapoptotic roles, and alleviate the ischemia and reperfusion damage. ${ }^{18-21}$ Studies that have examined the effect of levosimendan on organ functions have been scarce and controversial, and further studies are necessary to investigate this problem in Chinese elderly patients with sepsis. However, the current study found no evidence of any beneficial effect on the pulmonary, renal, hepatic, and coagulation functions, but confirmed that cardiovascular function was improved in the levosimendan group compared with that in the control group, and that the application of levosimendan improved cardiovascular function.

A recent study that investigated the effect of levosimendan at an infusion rate of $0.2-0.4 \mu \mathrm{g} / \mathrm{kg} / \mathrm{min}$ or with a loading dose did not reach a satisfactory conclusion. ${ }^{11}$ Peripheral vascular resistance and multiple organ perfusion excessively lowered by high-dose levosimendan are of no benefit to the improvement of organ functions and mortality rates in patients with sepsis. ${ }^{10}$ In the present study, levosimendan was administered through a continuous intravenous infusion of $0.1-0.2 \mu \mathrm{g} / \mathrm{kg} / \mathrm{min}$ without a loading dose rather than $0.2-0.4 \mu \mathrm{g} / \mathrm{kg} / \mathrm{min}$ with a loading dose. In clinical practice, the present study has demonstrated the significant benefit of smalldose levosimendan in maintaining stable hemodynamics, and the curative value of small-dose levosimendan for Chinese elderly patients with sepsis, suggesting that this kind of usage is appropriate for Chinese patients with sepsis.

\section{Conclusion}

In the present study, small-dose levosimendan could not reduce the mortality rates or enhance the respiratory, hepatic, renal, and coagulation functions, but succeeded in shortening the number of days of ICU and hospital stay and improving the cardiovascular function, which suggests that small-dose levosimendan is valuable for Chinese elderly patients with sepsis.

\section{Disclosure}

The authors report no conflicts of interest in this work.

\section{References}

1. Dellinger RP, Levy MM, Carlet JM, et al. Surviving Sepsis Campaign: international guidelines for management of severe sepsis and septic shock: 2008. Crit Care Med. 2008;36:296-327.

2. Singer M, Deutschman CS, Seymour CW, et al. The Third International Consensus Definitions for Sepsis and Septic Shock (Sepsis-3). JAMA. 2016;315:801-810.

3. Shankar-Hari M, Phillips GS, Levy ML, et al. Developing a new definition and assessing new clinical criteria for septic shock: for the Third International Consensus Definitions for Sepsis and Septic Shock (Sepsis-3). JAMA. 2016;315:775-787.

4. Nieminen MS, Fruhwald S, Heunks LM, et al. Levosimendan: current data, clinical use and future development. Heart Lung Vessel. 2013;5: $227-245$ 
5. Morelli A, Donati A, Ertmer C, et al. Levosimendan for resuscitating the microcirculation in patients with septic shock: a randomized controlled study. Crit Care. 2010;14:R232.

6. Shahul S, Gulati G, Hacker MR, et al. Detection of myocardial dysfunction in septic shock: a speckle-tracking echocardiography study. Anesth Analg. 2015;121:1547-1554.

7. Memiş D, Inal MT, Sut N. The effects of levosimendan vs dobutamine added to dopamine on liver functions assessed with noninvasive liver function monitoring in patients with septic shock. J Crit Care. 2012; 27:318.e1-e6.

8. Morelli A, Teboul JL, Maggiore SM, et al. Effects of levosimendan on right ventricular afterload in patients with acute respiratory distress syndrome: a pilot study. Crit Care Med. 2006;34:2287-2293.

9. Orme RM, Perkins GD, McAuley DF, et al. An efficacy and mechanism evaluation study of Levosimendan for the Prevention of Acute organ Dysfunction in Sepsis (LeoPARDS): protocol for a randomized controlled trial. Trials. 2014;15:199.

10. Fang M, Dong S. Effects of levosimendan on hemodynamics and cardiac function in patients with septic shock. Zhonghua Wei Zhong Bing Ji Jiu Yi Хие. 2014;26:692-696.

11. Gordon AC, Perkins GD, Singer M, et al. Levosimendan for the prevention of acute organ dysfunction in sepsis. N Engl J Med. 2016;375: 1638-1648.

12. Ferreira FL, Bota DP, Bross A, Melot C, Vincent JL. Serial evaluation of the SOFA score to predict outcome in critically ill patients. JAMA. 2001;286:1754-1758.

13. Gordon AC, Mason AJ, Thirunavukkarasu N, et al. Effect of early vasopressin vs norepinephrine on kidney failure in patients with septic shock: the VANISH randomized clinical trial. JAMA. 2016;316: 509-518.
14. Gordon AC, Mason AJ, Perkins GD, et al. The interaction of vasopressin and corticosteroids in septic shock: a pilot randomized controlled trial. Crit Care Med. 2014;42:1325-1333.

15. Zhang D, Yao Y, Qian J, Huang J. Levosimendan improves clinical outcomes of refractory heart failure in elderly Chinese patients. Med Sci Monit. 2015;21:2439-2445.

16. Ukkonen H, Saraste M, Akkila J, et al. Myocardial efficiency during calcium sensitization with levosimendan: a noninvasive study with positron emission tomography and echocardiography in healthy volunteers. Clin Pharmacol Ther. 1997;61:596-607.

17. Zangrillo A, Putzu A, Monaco F, et al. Levosimendan reduces mortality in patients with severe sepsis and septic shock: a meta-analysis of randomized trials. J Crit Care. 2015;30:908-913.

18. Wang Q, Yokoo H, Takashina M, et al. Anti-inflammatory profile of levosimendan in cecal ligation-induced septic miceand in lipopolysaccharide-stimulated macrophages. Crit Care Med. 2015;43: e508-e520.

19. Hasslacher J, Bijuklic K, Bertocchi C, et al. Levosimendan inhibits release of reactive oxygen species in polymorphonuclear leukocytes in vitro and in patients with acute heart failure and septic shock: a prospective observational study. Crit Care. 2011;15:R166.

20. Parissis JT, Adamopoulos S, Antoniades C, et al. Effects of levosimendan on circulating pro-inflammatory cytokines and soluble apoptosis mediators in patients with decompensated advanced heart failure. Am J Cardiol. 2004;93:1309-1312.

21. du Toit EF, Genis A, Opie LH, Pollesello P, Lochner A. A role for the RISK pathway and K(ATP) channels in pre- and post-conditioning induced by levosimendan in the isolated guinea pig heart. $\mathrm{Br} J$ Pharmacol. 2008;154:41-50.
Clinical Interventions in Aging

\section{Publish your work in this journal}

Clinical Interventions in Aging is an international, peer-reviewed journal focusing on evidence-based reports on the value or lack thereof of treatments intended to prevent or delay the onset of maladaptive correlates of aging in human beings. This journal is indexed on PubMed Central, MedLine,

\section{Dovepress}

CAS, Scopus and the Elsevier Bibliographic databases. The manuscript management system is completely online and includes a very quick and fair peer-review system, which is all easy to use. Visit http://www.dovepress. com/testimonials.php to read real quotes from published authors. 